\title{
Triptolide inhibits JAK2/STAT3 signaling and induces lethal autophagy through ROS generation in cisplatin-resistant SKOV3/DDP ovarian cancer cells
}

\author{
YANYING ZHONG ${ }^{1}$, FUYIN LE $^{1}$, JIAO CHENG $^{2}$, CHEN LUO $^{3}$, XIALI ZHANG ${ }^{4}$, \\ XINGWU WU ${ }^{5}$, FANG XU ${ }^{6}$, QI ZUO ${ }^{1}$ and BUZHEN TAN ${ }^{1}$ \\ ${ }^{1}$ Department of Obstetrics and Gynecology, The Second Affiliated Hospital of Nanchang University; \\ ${ }^{2}$ Department of Tumour Immunology, School of Basic Medicine Sciences, Nanchang University Medical College; \\ ${ }^{3}$ Department of General Surgery, The Second Affiliated Hospital of Nanchang University; \\ ${ }^{4}$ Department of Laboratory Animal Science, Nanchang University; ${ }^{5}$ Jiangxi Maternal and \\ Child Health Hospital, Nanchang, Jiangxi 330006; ${ }^{6}$ Department of Obstetrics and Gynecology, \\ The Third Hospital of Nanchang University, Nanchang, Jiangxi 330009, P.R. China
}

Received November 16, 2020; Accepted March 4, 2021

DOI: $10.3892 / o r .2021 .8020$

\begin{abstract}
Advanced and recurrent ovarian cancer has a poor prognosis and is frequently resistant to numerous therapeutics; thus, safe and effective drugs are needed to combat this disease.Previous studies have demonstrated that triptolide (TPL) exhibits anticancer and sensitization effects against cisplatin (DDP)-resistant ovarian cancer both in vitro and in vivo by inducing apoptosis; however, the involvement of autophagy induced by TPL in resistant ovarian carcinoma remains unclear. In the present study, the results revealed that TPL induced autophagy to facilitate SKOV3/DDP ovarian cancer cell death. The xenograft experiment revealed that the autophagy inhibitor CQ significantly reduced TPL-mediated chemosensitization and tumor growth inhibition. Mechanically, TPL-induced autophagy in SKOV3/DDP cells was associated with the induction of ROS generation and inhibition of the Janus kinase 2 (JAK2)/signal transducer and activator of
\end{abstract}

Correspondence to: Professor Buzhen Tan, Department of Obstetrics and Gynecology, The Second Affiliated Hospital of Nanchang University, 1 Minde Road, Nanchang, Jiangxi 330006, P.R. China

E-mail: tanbuzhen@sina.com

Abbreviations: TPL, triptolide; DDP, cisplatin; 3-MA, 3-methyladenine; CQ, chloroquine; NAC, N-acetyl-1-cysteine; ROS, reactive oxygen species; JAKs, Janus family kinases; STAT3, signal transducer and activator of transcription-3; Mcl-1, myeloid cell leukemia-1; FBS, fetal bovine serum; CCK-8, Cell Count Kit-8; OD, optical density; SDS-PAGE, sodium dodecyl sulfate-polyacrylamide gel electrophoresis; PVDF, polyvinylidene difluoride; IP, immunoprecipitation; SPF, Specific-Pathogen-Free; SD, standard deviation

Key words: triptolide, ovarian cancer, autophagy, reactive oxygen species, JAK2/STAT3 pathway transcription-3 (STAT3) pathway. The inhibitory effect of TPL on the JAK2/STAT3 pathway could be restored in the presence of the antioxidant NAC. Furthermore, it was further determined that TPL disrupted the interaction between Mcl-1 and Beclin1, which was prevented by the JAK2/STAT3 signaling activator IL-6. Overall, the present results revealed a novel molecular mechanism whereby TPL induced lethal autophagy through the ROS-JAK2/STAT3 signaling cascade in SKOV3/DDP cells. The present study has provided the groundwork for future application of TPL in the treatment of ovarian cancer.

\section{Introduction}

Epithelial ovarian cancer is the second most prevalent gynecological malignancy with a considerably high mortality rate (1). Unfortunately, most patients are usually diagnosed at an advanced stage due to a lack of effective screening strategies (2). Currently, surgical resection and platinum-based combination chemotherapy are the principal treatments for this disease. Despite great progress in ovarian cancer treatment, the majority of patients eventually experience chemoresistance, which could lead to tumor recurrence and metastasis $(3,4)$. Therefore, the search for effective therapies remains to be resolved.

Triptolide (TPL), a diterpene triepoxide extracted from the Chinese herb Tripterygium wilfordii, has been reported to present marked effects in various cancers, such as hepatocellular (5) and pancreatic carcinoma (6), bladder (7), breast (8) and ovarian cancers (9-13). The anticancer properties of TPL have been revealed in numerous contexts, including the induction of cell cycle arrest and apoptosis and the inhibition of cell proliferation, metastasis and angiogenesis (5-13). Our previous study demonstrated that TPL synergistically inhibited the growth of cisplatin (DDP)-resistant ovarian cancer cells by reactive oxygen species (ROS)-mediated apoptosis induction (12). Recently, TPL has been considered to be a potential autophagy modulator (14). However, the autophagic effect of TPL on resistant human epithelial ovarian cancer cells is not clear. 
Autophagy is a highly conserved cellular catabolic pathway that plays a homeostatic role in normal cells. There is a growing interest in utilizing autophagic mechanisms to suppress tumor growth (15). Intriguingly, autophagy has multifaceted and context-specific roles in cancer. Altered autophagic activity has been revealed to favor cancer cell survival in response to stress stimuli, such as starvation, ROS, and chemotherapy, and mediate resistance to these treatments (16). On the other hand, excessive autophagy is known as type II programmed cell death and has been exploited as a potential strategy for cancer therapy and tumor chemosensitization, especially in apoptosis-defective tumor cells (17). The regulation of autophagy is highly complex. Notably, signal transducer and activator of transcription-3 (STAT3) is a redox-sensitive transcription factor that participates in the regulation of autophagic processes upon activation by Janus family kinases (JAKs) $(18,19)$. Activated STAT3 dimerizes, transfers into the nucleus and regulates the transcription of numerous target genes, including certain autophagy-related genes (20). To date, accumulating evidence and studies have established the role of the aberrantly active Janus kinase 2 (JAK2)/STAT3 pathway in ovarian cancer progression and chemoresistance $(21,22)$. Inhibition of the JAK2/STAT3 pathway may shed new light on cancer treatment and provide new targets for ovarian cancer therapy (21).

In the present study, the autophagic role of TPL in DDP-resistant ovarian cancer was evaluated using SKOV3/DDP cells and the effects of TPL-induced autophagy on chemosensitization in vivo were further studied. In addition, systematic research on the underlying mechanisms involved in TPL-induced autophagy against SKOV3/DDP cells was performed in vitro, by various molecular biological techniques, such as flow cytometry, gene silencing, western blotting, immunofluorescence staining and co-immunoprecipitation.

\section{Materials and methods}

Antibodies and reagents. Antibodies to phosphorylated (p)-JAK2 (product no. 3776; 1:1,000), JAK2 (product no. 3230; 1:1,000), p-STAT3 (Y705) (product no. 9145; used at 1:1,000 for western blotting and 1:100 for immunofluorescence staining), STAT3 (product no. 12640; 1:1,000), myeloid cell leukemia-1 (Mcl-1; product no. 94296; 1:1,000), LC3 (product no. 4599; used at 1:1,000 for western blotting and 1:6,400 for immunohistochemistry), p62 (product no. 88588; used at 1:1,000 for western blotting and 1:1,600 for immunohistochemistry), Beclin1 (product no. 3495; 1:1,000), caspase-3 (product no. 9662; used at 1:1,000 for western blotting and immunohistochemistry), $\beta$-actin (product no. 3700; 1:1,000) and Ki67 (product no. 12202; 1:800) were purchased from Cell Signaling Technology, Inc. HRP-labeled anti-rabbit (cat. no. SA00001-2; 1:5,000) and anti-mouse IgG (cat. no. SA00001-1; 1:5,000) were obtained from ProteinTech, Group, Inc. Chemicals, including triptolide (TPL), chloroquine (CQ), 3-methyladenine (3-MA), N-acetyl-L-cysteine (NAC), cisplatin (DDP), interleukin (IL)-6 and AG490 were purchased from Sigma-Aldrich; Merck KGaA. Cell Counting Kit-8 (CCK-8) assay and Reactive Oxygen Species (ROS) Assay Kit were obtained from Beyotime Institute of Biotechnology.

Cell culture. Cisplatin-resistant SKOV3/DDP and parental SKOV3 cells were purchased from China Center for Type Culture
Collection and preserved at the Key Laboratory of Molecular Center of Jiangxi Province. The cells were routinely cultured in RPMI-1640 medium containing fetal bovine serum (FBS; $10 \%)$, penicillin/streptomycin $(100 \mathrm{U} / \mathrm{ml})$ in a $5 \%$ humidified $\mathrm{CO}_{2}$ atmosphere at $37^{\circ} \mathrm{C}$. Cisplatin $(0.3 \mu \mathrm{g} / \mathrm{ml})$ was added to the SKOV3/DDP culture media to maintain its acquired resistance.

Autophagy flux analysis. To track autophagic flux, SKOV3/DDP cells were transfected with mRFP-GFP-LC3 adenovirus (Hanbio Biotechnology Co., Ltd.) following the manufacturer's recommendations and performed as previously described (23). Briefly, SKOV3/DDP cells $\left(1 \times 10^{6}\right.$ cells $\left./ \mathrm{ml}\right)$ were treated with TPL $(0,25,50$ and $100 \mathrm{nM})$ for $12 \mathrm{~h}$, or treated with AG490 $(50 \mu \mathrm{M})$ for $24 \mathrm{~h}$ at $37^{\circ} \mathrm{C}$, respectively. After drug treatment, the formation of autophagosomes (yellow puncta) and autolysosomes (red puncta) were detected using a confocal laser scanning microscope under an x400 magnification.

Cell viability assay. Cell viability was measured using Cell Counting Kit-8 (CCK-8) assay. Briefly, SKOV3/DDP cells $\left(1 \times 10^{4}\right.$ cells/well $)$ were treated with TPL (100 nM), CQ $(10 \mu \mathrm{M}), 3-\mathrm{MA}(10 \mathrm{mM})$ or a combination of these compounds for $24 \mathrm{~h}$ at $37^{\circ} \mathrm{C}$. Following treatment, CCK-8 solution was added and incubated at $37^{\circ} \mathrm{C}$ for $4 \mathrm{~h}$. The optical density (OD) value of each well was measured at $450 \mathrm{~nm}$ using a Microplate Reader (Molecular Devices, LLC), and the cell viability was then calculated.

Western blot analysis. Immunoblotting analyses were performed as previously described (12). The whole cell protein extracts were prepared using the RIPA lysis buffer (Solarbio Life Science), and the protein concentrations were quantified with BCA Protein Assay Kit. Equal amounts of protein (30 $\mu \mathrm{g} /$ lane) were separated on $10-15 \%$ sodium dodecyl sulfate-polyacrylamide gel electrophoresis (SDS-PAGE) and transferred to a polyvinylidene difluoride (PVDF) membrane (Thermo Fisher Scientific, Inc.). Following blocking for $2 \mathrm{~h}$ using $5 \%$ non-fat milk at room temperature, the membrane was incubated overnight with the specific primary antibodies against p-JAK2, JAK2, p-STAT3 (Y705), STAT3, Mcl-1, LC3, p62, Beclin1, caspase-3, and the internal control $\beta$-actin at $4^{\circ} \mathrm{C}$. After washing with PBS, the membranes were incubated with the corresponding horseradish peroxidase (HRP)-conjugated goat anti-rabbit IgG or anti-mouse $\operatorname{IgG}$ at room temperature for $1 \mathrm{~h}$. The blot bands were detected using an enhanced chemiluminescence (ECL) kit (Thermo Fisher Scientific, Inc.), and imaged and quantified using the ChemiDoc XRS System (Bio-Rad Laboratories, Inc.).

Measurement of intracellular ROS. Reactive Oxygen Species Assay Kit was applied to measure the level of intracellular ROS in SKOV3/DDP cells. Briefly, SKOV3/DDP cells $\left(1 \times 10^{6}\right.$ cells $\left./ \mathrm{ml}\right)$ were treated with TPL $(100 \mathrm{nM})$ alone or in presence of NAC $(5 \mathrm{mM})$ at $37^{\circ} \mathrm{C}$ for $12 \mathrm{~h}$, then washed three times in PBS and incubated with DCFH-DA at $37^{\circ} \mathrm{C}$ for $20 \mathrm{~min}$ in the dark. The DCF fluorescence intensity was examined and analyzed by cell flow cytometry (FACSCalibur; BD Biosciences).

Immunofluorescence staining. Immunofluorescence staining was carried out to analyze the expression of p-STAT3 (Y705) in SKOV3/DDP cells. Briefly, SKOV3/DDP cells $\left(1 \times 10^{6}\right.$ cells $\left./ \mathrm{ml}\right)$ 
were treated with or without $100 \mathrm{nM}$ TPL at $37^{\circ} \mathrm{C}$ for $24 \mathrm{~h}$, and then fixed with $4 \%$ paraformaldehyde for $20 \mathrm{~min}$ on ice and permeablized with $0.5 \%$ Triton $\mathrm{X}-100$ for $10 \mathrm{~min}$ at $37^{\circ} \mathrm{C}$. Then, the cells were incubated with anti-p-STAT3 (Y705) antibody at $4^{\circ} \mathrm{C}$ overnight and subsequently with CoraLite 488 -conjugated Affinipure goat anti-rabbit IgG secondary antibody (cat. no. SA00013-2; 1:500; ProteinTech Group, Inc.) for $1 \mathrm{~h}$ at room temperature in the dark. Finally, the cells were incubated with DAPI at room temperature for $10 \mathrm{~min}$ to stain nuclei, and the and the images were obtained using a confocal microscope (Olympus FV1000; Olympus Corporation) at a magnification of $\mathrm{x} 400$.

Co-immunoprecipitation (Co-IP) assay. Co-IP assay was performed as previously reported (24). Briefly, 1,000 $\mu \mathrm{g}$ of whole cell lysates were prepared with $1 \mathrm{ml}$ of IP lysis buffer (cat. no. 87787; Pierce IP Lysis Buffer; Thermo Fisher Scientific, Inc.) after drug treatment. Then the lysate was incubated with the antibody against Beclin1 and $100 \mu \mathrm{l}$ of Protein A/G magnetic beads (cat. no. 88803; Pierce; Thermo Fisher Scientific, Inc.) at $4^{\circ} \mathrm{C}$ overnight on a rocking platform. The immunoprecipitated pellets were collected by centrifugation at $12,000 \mathrm{x} \mathrm{g}$ for $5 \mathrm{~min}$ at $4^{\circ} \mathrm{C}$. Then the pellets were washed three times with the cold lysis buffer, boiled in 2X SDS loading buffer and analyzed by western blot analysis with anti-Mcl-1.

Beclin1 siRNA transfection. After SKOV3/DDP cells were grown into 6-well plates and reached $50 \%$ confluence, cells were transiently transfected with the Beclin1 small interfering (si)RNA or negative control siRNA (100 pmol) using Lipofectamine 2000 ((Invitrogen; Thermo Fisher Scientific, Inc.) according to the manufacturer's instructions. Briefly, $5 \mu \mathrm{l}$ (100 pmol) Beclin1 siRNA and $5 \mu 1$ Lipofectamine 2000 were diluted in $50 \mu \mathrm{l}$ RPMI-1640 medium, respectively, and were incubated for $5 \mathrm{~min}$ at room temperature. Then, the diluted Beclin1 siRNA and Lipofectamine 2000 were mixed and incubated for $15 \mathrm{~min}$ at room temperature. Next, the complexes were added to cells and incubated for $6 \mathrm{~h}$ at $37^{\circ} \mathrm{C}$ in a $5 \% \mathrm{CO}_{2}$ incubator. Thereafter, the cells were washed with PBS and suspended in the RPMI-1640 medium plus $10 \%$ FBS. After 48 h of transfection, cells were treated or untreated with $100 \mathrm{nM}$ TPL for an additional $24 \mathrm{~h}$ and collected for western blot analysis. The Beclin1 siRNA (5'-GGA TGACAGTGAACAGTTA-3') and negative control siRNA (5'-UUCUCCGAACGUGUCACGUTT-3') were designed and synthesized by Guangzhou Ribobio Co., Ltd.

Ectopic expression of Mcl-1 by transient transfection. Proliferating SKOV3/DDP cells in a 6-well plate were transiently transfected with the pcDNA3.1-3xFLAG vector (GV141; Shanghai Genechem, Co., Ltd.) encoding Mcl-1 or the empty vector $(1 \mu \mathrm{g} / \mu \mathrm{l})$ at $50 \%$ confluence using Lipofectamine 3000 (Invitrogen; Thermo Fisher Scientific, Inc.) according to the manufacturer's instructions. Briefly, $5 \mu \mathrm{l}(1 \mu \mathrm{g} / \mu \mathrm{l})$ Mcl-1-vector and $10 \mu$ l Lipofectamine 3000 were diluted in $125 \mu \mathrm{l}$ RPMI-1640 medium, respectively, and were incubated for $5 \mathrm{~min}$ at room temperature. Then, the diluted Mcl-1-vector and Lipofectamine 3000 mixture were maintained at room temperature for $15 \mathrm{~min}$. Next, the complexes were added to each well and incubated for $6 \mathrm{~h}$ at $37^{\circ} \mathrm{C}$. Subsequently, the cells were washed with PBS and replaced with RPMI-1640 medium containing $10 \%$ FBS. After $48 \mathrm{~h}$ of transfection, cells were treated or untreated with $100 \mathrm{nM}$ TPL for an additional $24 \mathrm{~h}$ and harvested for western blot analysis.

In vivo xenograft study. The female athymic BALB/CA-u nude mice (4-6 weeks old; $18 \pm 2 \mathrm{~g} ; \mathrm{n}=25$ ) were purchased from Hunan SJA Laboratory Animal Co., Ltd. and housed at the SPF-level laboratory animal center in Nanchang University (26-28 ${ }^{\circ}$; $55 \%$ humidity; 10-h light/14-h dark cycle). All mice had free access to sterilized food and water. All animal experiments were approved by the Ethics Committee of Nanchang University and performed in compliance with the approved guidelines. A tumor xenograft was established in a murine model as described in our previous study $(11,13)$. Briefly, single-tumor cells of SKOV3/DDP suspensions $\left(5 \times 10^{6}\right)$ were subcutaneously injected into the right armpit of each BALB/CA-u nude mice. Seven days after cell implantation, the mice bearing tumors were randomized into five groups ( $n=5 /$ group) and initially treated as follows: i) Vehicle group $(50 \mathrm{ml} / \mathrm{kg} /$ day PBS every day, i.p.); ii) TPL group $(0.15 \mathrm{mg} / \mathrm{kg} /$ day TPL every day, i.p.); iii) DDP group (4 mg/kg/day DDP on the $1 \mathrm{st}$ and 8th days, i.p.); iv) TPL + DDP group $(0.15 \mathrm{mg} / \mathrm{kg} /$ day TPL every day, $4 \mathrm{mg} / \mathrm{kg} /$ day DDP on the $1 \mathrm{st}$ and 8 th days, i.p.); and v) TPL + DDP + CQ group $(0.15 \mathrm{mg} / \mathrm{kg} /$ day TPL every day, $4 \mathrm{mg} / \mathrm{kg} /$ day DDP on the $1 \mathrm{st}$ and 8 th days, $25 \mathrm{mg} / \mathrm{kg} /$ day CQ every day, i.p.). All drugs were intraperitoneally (i.p.) injected into the mice as aforementioned (day 0 was considered as the beginning day of drug treatment). Tumor volumes and body weight were verified every other day. The tumor volume was calculated using the standard formula: Volume $\left(\mathrm{mm}^{3}\right)=$ width $^{2}\left(\mathrm{~mm}^{2}\right) \times$ length $(\mathrm{mm}) \times 0.5$. After treatment for 10 days, all the mice were euthanized. The tumors were harvested, weighed and subjected to further immunohistochemical analysis as previously described $(11,13)$.

Statistical analysis. All experiments were performed at least three times, and the results are presented as the mean \pm standard deviation (SD). Statistical analyses were performed using GraphPad Prism version 6.0 software (GraphPad Software, Inc.).

Unpaired Student's t-test was employed for comparisons of means between two groups, and one-way ANOVA with Tukey's post hoc test were employed for the comparison of multiple groups. The criterion for statistical significance was indicated as $\mathrm{P}<0.05$.

\section{Results}

TPL treatment triggers autophagic cell death in SKOV3/DDP cells. TPL has been revealed to stimulate autophagy in some cancer cells (25-28), while the effect of TPL on autophagy regulation in drug-resistant ovarian cancer cells has not been reported. Therefore, mRFP-GFP tandem fluorescent-tagged LC3 was first used to track autophagic flux in SKOV3/DDP cells. As revealed in Fig. 1A, a significant increase in the number of autophagosomes (yellow puncta) and autolysosomes (red puncta) were observed following TPL treatment under a confocal microscope. Next, the expression of two specific autophagy markers, the proteins LC3 and p62 were assessed. Western blot analysis revealed that TPL treatment markedly increased the ratio of LC3-II/LC3-I but reduced p62 


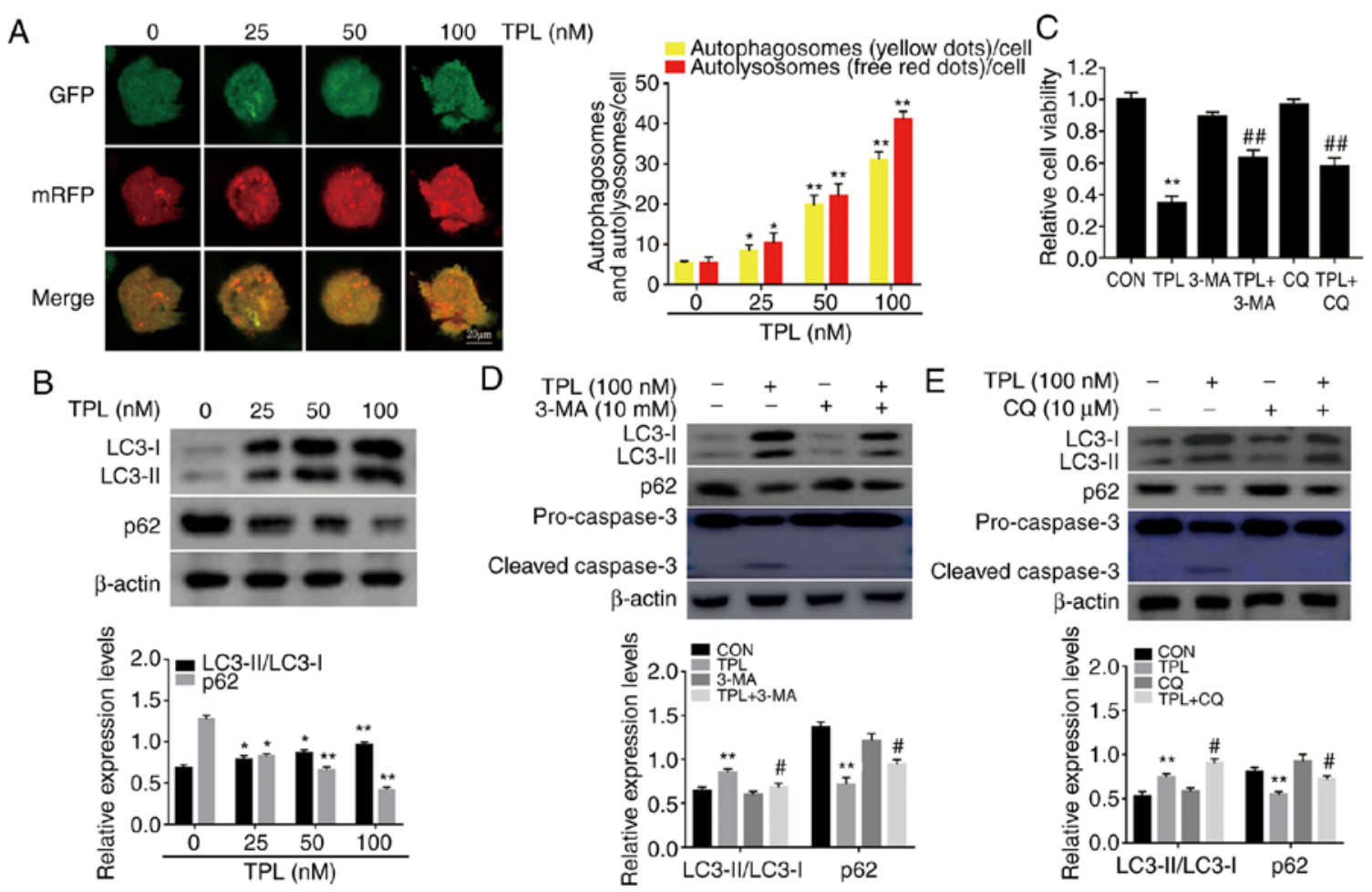

Figure 1. TPL treatment induces autophagic cell death in DDP-resistant ovarian cancer SKOV3/DDP cells. (A) SKOV3/DDP cells transfected with mRFP-GFP-LC3 adenovirus were treated with the indicated doses of TPL for $12 \mathrm{~h}$, and then analyzed using a confocal microscope (magnification, x400). Representative images respectively revealed the autophagosomes (yellow puncta) and the autolysosomes (red puncta). Scale bar, $20 \mu \mathrm{m}$. ${ }^{*} \mathrm{P}<0.05$ and ${ }^{* *} \mathrm{P}<0.01 \mathrm{vs}$. the control (untreated) group. (B) SKOV3/DDP cells treated with indicated concentrations of TPL for $24 \mathrm{~h}$ were subjected to immunoblotting for the levels of LC3 and p62. (C-E) SKOV3/DDP cells were pre-incubated with or without autophagy inhibitor 3-MA $(10 \mathrm{mM})$ or CQ $(10 \mu \mathrm{M})$ for $1 \mathrm{~h}$, followed by $24-\mathrm{h} \mathrm{TPL}(100 \mathrm{nM})$ treatment. (C) TPL cytotoxicity was detected by CCK-8 assay. (D and E) LC3, p62 and cleaved caspase-3 levels were examined by western blot analysis. ${ }^{*} \mathrm{P}<0.05$ and ${ }^{* * *} \mathrm{P}<0.01$ vs. the Con group. ${ }^{\#} \mathrm{P}<0.05$ and ${ }^{\# \#} \mathrm{P}<0.01$ vs. the TPL group. TPL, triptolide; DDP, cisplatin; 3-MA, 3-methyladenine; CQ, chloroquine; Con, control.

expression in a dose-dependent manner in SKOV3/DDP cells (Fig. 1B). These results clearly revealed that TPL treatment enhanced autophagic flux in ovarian cancer cells, which was consistent with previous studies (25-28).

Our group previously revealed that TPL induced apoptosis-mediated cell death in DDP-resistant ovarian cancer cells $(9,10,12,13)$. Given the dual role of autophagy in cancer cell death, widely used autophagy inhibitors (3-MA and CQ) were applied to further confirm whether this autophagic response mediates the cytotoxic effects of TPL. As revealed in Fig. 1C, cell viability was significantly restored following treatment with TPL in the presence of 3-MA or CQ. Moreover, the expression of cleaved caspase- 3 was also significantly suppressed when TPL-induced autophagy was inhibited by 3-MA or CQ (Fig. 1D and E). These data indicated that autophagy contributed to TPL-induced SKOV3/DDP cell death. The autophagic effect of TPL on parental SKOV3 cells was then evaluated. Consistent with the effect observed in SKOV3/DDP cells, TPL also prompted autophagy of SKOV3 cells (Fig. S1A). In addition, it was also revealed that the cytotoxic effect of TPL on SKOV3 cells was significantly reduced by pretreatment with 3-MA (Fig. S1B). Thus, the data aforementioned suggested that the autophagy induced by TPL was cytotoxic to ovarian cancer cells, rather than cytoprotective.

TPL potentiates the antitumor effect of DDP in vivo partially via autophagy induction. Our group previously demonstrated that TPL could enhance the suppressive effects of DDP on tumor growth in a SKOV3/DDP xenograft model $(11,13)$. To further evaluate the effects of TPL-induced autophagy on chemosensitization in vivo, the impact of the combination of $\mathrm{TPL}+\mathrm{DDP}+\mathrm{CQ}$ on the growth of SKOV3/DDP xenografts was determined. Mice-bearing SKOV3/DDP cells were randomly separated into five groups: Vehicle, TPL, DDP, TPL + DDP, and TPL + DDP + CQ. All animals tolerated TPL treatment well. The mice did not exhibit any obvious change in body weight (Fig. 2A). The tumor volume and tumor weight in the TPL + DDP group were significantly reduced compared to those in the TPL + DDP + CQ group or any other single treatment group (Fig. $2 \mathrm{~B}$ and $\mathrm{C}$ ). In agreement with this observation, proliferation-related $\mathrm{Ki}-67$ (the brown color area) was greatly downregulated in the TPL + DDP group (Fig. 2D). Additionally, the immunohistochemical staining results also revealed that autophagy inhibition significantly reduced the chemosensitization effect of TPL on SKOV3/DDP cells in vivo (Fig. 2D). It was concluded from these results that the chemosensitization effect of TPL was dependent on autophagy.

TPL-induced SKOV3/DDP cell autophagy is closely related to intracellular ROS generation. Then, systematic research on the underlying mechanisms involved in TPL-induced autophagy against SKOV3/DDP cells was performed in vitro. ROS have been documented as common mediators of cell apoptosis and autophagy (29). In a previous study by our group, it was found that TPL induced ROS-related apoptosis in DDP-resistant ovarian cancer cells (12). Therefore, DCFH-DA was first used 

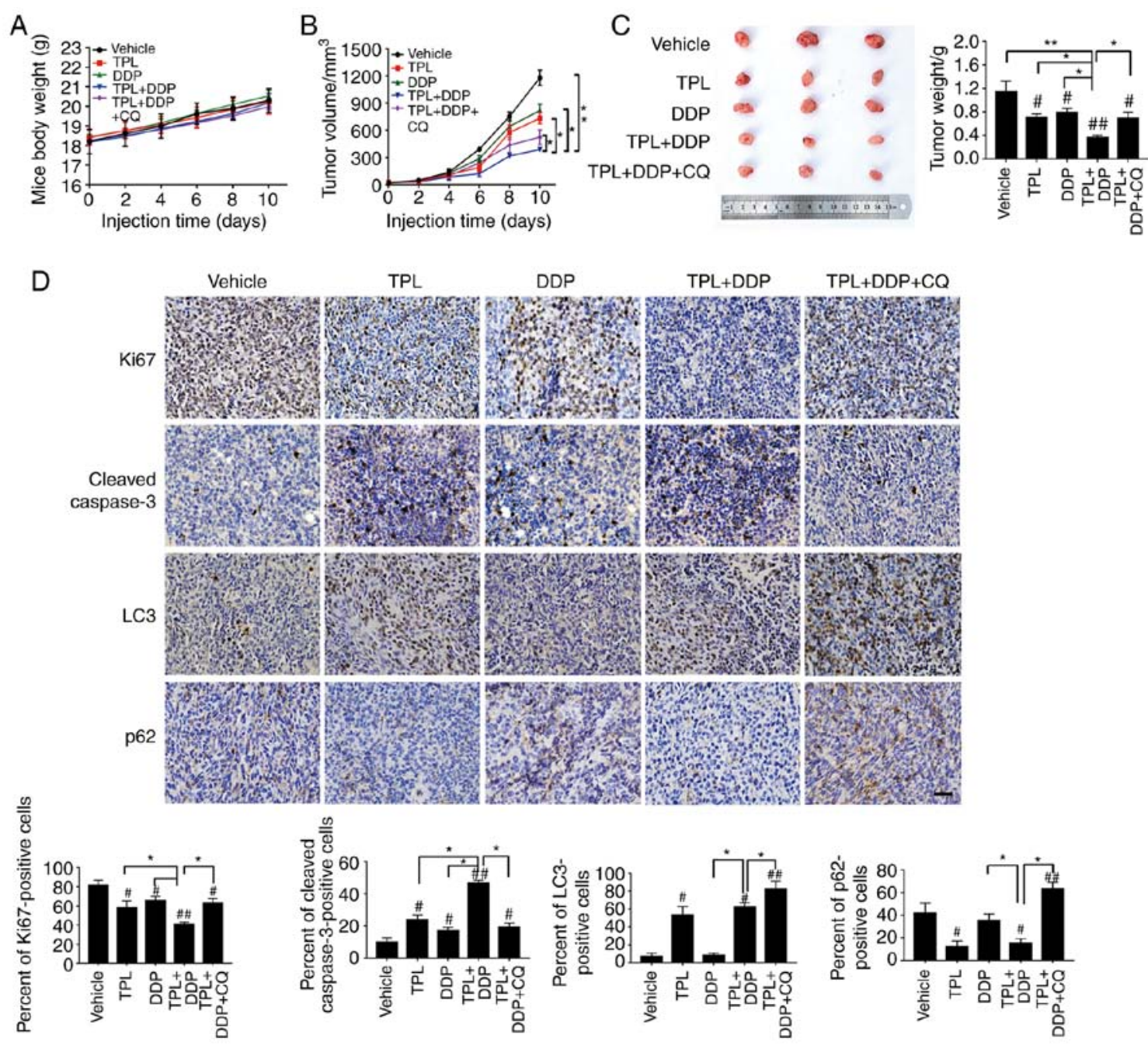

Figure 2. TPL potentiates the antitumor effect of DDP through autophagy induction in vivo. Mice bearing SKOV3/DDP tumor cells were intraperitoneally injected with PBS, TPL, DDP, TPL + DDP, and TPL + DDP + CQ. (A) The body weight of mice and (B) tumor volume were measured every two days during the administration period. At the end of experiment, tumors were removed, photographed and (C) weighed. (D) Immunohistochemical analysis (Ki67, cleaved caspase-3, LC3 and p62 staining) of tumor tissue sections isolated from the indicated groups of mice (magnification, $\mathrm{x} 400$ ). Scale bar, $100 \mu \mathrm{m}$. ${ }^{*} \mathrm{P}<0.05$ and ${ }^{* *} \mathrm{P}<0.01$ vs. the TPL + DDP group. ${ }^{\#} \mathrm{P}<0.05$ and ${ }^{\# \#} \mathrm{P}<0.01$ vs. the Vehicle group. TPL, triptolide; DDP, cisplatin; CQ, chloroquine; Con, control.

as a probe to examine the intracellular ROS levels. As revealed in Fig. 3A and B, the mean DCF fluorescence intensity of SKOV3/DDP cells was significantly increased in response to $100 \mathrm{nM}$ of TPL treatment, as compared with the control group. Additionally, this increase was significantly attenuated when the cells were pretreated with the well-known antioxidant NAC (Fig. 3A and B). These data indicated that TPL promoted the generation of cellular ROS in SKOV3/DDP cells. To evaluate the role of ROS in TPL-induced autophagy, SKOV3/DDP cells were incubated with TPL in the presence or absence of NAC. Notably, pretreatment with NAC strongly reduced the number of autophagosomes and autolysosomes compared with TPL treatment alone (Fig. 3C). Consistently, NAC strongly blocked the TPL-induced upregulation of LC3-II/LC3-I expression levels while enhancing p62 expression (Fig. 3D). Overall, these findings suggested that the autophagic inducing effect of TPL on SKOV3/DDP cells was associated with ROS generation.

ROS generation is upstream of JAK2/STAT3 pathway inactivation in TPL-induced autophagy. The JAK2/STAT3 pathway is constitutively activated in ovarian cancer $(21,22)$, has a well-established role in autophagy regulation and can be influenced by ROS (30). First, to evaluate the association between activation of the JAK2/STAT3 pathway and autophagy in SKOV3/DDP cells, AG490 (a JAK2/STAT3 signaling inhibitor) was used. As revealed in Fig. $4 \mathrm{~A}$ and B, the level of autophagy significantly increased after treatment with AG490, suggesting that inhibition of the JAK2/STAT3 pathway may play an important role in autophagy induction.

The effect of TPL treatment on the JAK2/STAT3 signaling pathway was next examined. Western blot analysis revealed that TPL treatment led to noticeable inhibition of p-JAK2 and p-STAT3 (Y705) (Fig. 4C). Additionally, immunofluorescence analysis revealed that TPL incubation significantly decreased the nuclear level of p-STAT3 (Y705) (Fig. 4D). These results indicated that TPL treatment inhibited the JAK2/STAT3 pathway in SKOV3/DDP cells.

To further reveal the importance of the JAK2/STAT3 pathway in modulating TPL-induced autophagy, SKOV3/DDP cells were pretreated with IL-6 (a JAK2/STAT3 signaling activator). It was revealed that IL- 6 strongly attenuated the LC 3 conversion and p62 degradation accompanied by the 

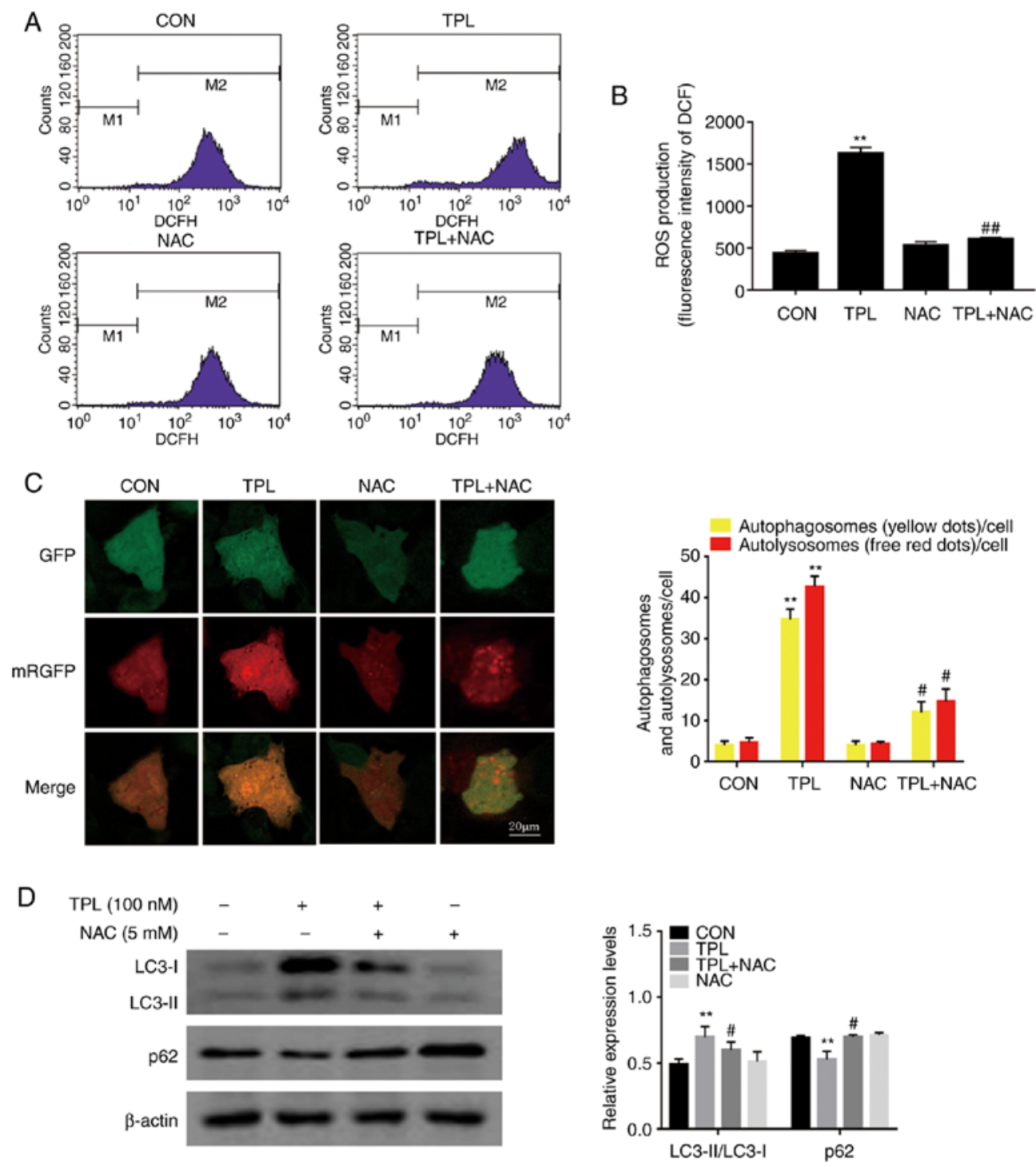

Figure 3. TPL-triggered autophagy in SKOV3/DDP cells is ROS-dependent. SKOV3/DDP cells were treated with NAC (5 mM) for $1 \mathrm{~h}$ prior to TPL (100 $\mathrm{nM}$ ) incubation, and then the following experiments were carried out. (A and B) The intracellular ROS level was analyzed using DCFH-DA labeling by flow cytometry. Data are expressed as the mean \pm SD of three independent experiments. (C) Autophagosomes and autolysosomes were tracked by confocal microscope using mRFP-GFP tandem fluorescent-tagged LC3. Scale bar, $20 \mu \mathrm{m}$. (D) Autophagy marker proteins (LC3 and p62) were assessed using immunoblotting analysis. ${ }^{* *} \mathrm{P}<0.01$ vs. the Con group. ${ }^{*} \mathrm{P}<0.05$ and ${ }^{\# \#} \mathrm{P}<0.01$ vs. the TPL group. TPL, triptolide; DDP, cisplatin; ROS, reactive oxygen species; NAC, $\mathrm{N}$-acetyl-1-cysteine; Con, control.

upregulation of p-JAK2 and p-STAT3 (Y705) compared to those of the TPL group (Fig. 4E). These data indicated that TPL triggered autophagy in SKOV3/DDP cells at least partially by modulating JAK2/STAT3 signaling. Notably, the TPL-induced reductions in p-JAK2 and p-STAT3 (Y705) could be restored in the presence of NAC (Fig. 4F). Thus, ROS-mediated inhibition of the JAK2/STAT3 signaling pathway was involved in TPL-induced autophagy.

JAK2/STAT3 pathwayinactivation contributes to TPL-induced autophagy by regulating the Beclin1/Mcl-1 interaction. Next, the downstream signaling molecules of STAT3 involved in the regulation of TPL-induced autophagy were investigated. It has been reported that Mcl-1, a well-known antiapoptotic protein that is transcriptionally regulated by STAT3, negatively regulates autophagy by binding to Beclin1 $(24,31)$. Therefore, the protein levels of Mcl-1 and Beclin1 were analyzed and it was revealed that TPL efficiently decreased Mcl-1 levels and increased Beclin1 protein levels in a dose-dependent manner (Fig. 4C). Next, an immunoprecipitation assay was conducted to monitor the interaction between Beclin1 and Mcl-1 in SKOV3/DDP cells. It was revealed that Mcl-1 and Beclin1 immunoprecipitated with each other in SKOV3/DDP cells under basal conditions (Fig. 5A), whereas the interaction markedly decreased in the presence of TPL (Fig. 5B).

Notably, IL-6 significantly prevented TPL-mediated Mcl-1 downregulation (Fig. 4E) and the Beclin1/Mcl-1 interaction (Fig. 5B). Furthermore, transient overexpression of Mcl-1 (Fig. 5C) and siRNA-mediated Beclin1 downregulation (Fig. 5D) significantly attenuated LC3-II accumulation and p62 degradation induced by TPL. Collectively, these results indicated that inhibition of the JAK2/STAT3/Mcl-1 pathway and the subsequent disruption of the Beclin1/Mcl-1 interaction are vital to TPL-induced autophagy. 
A

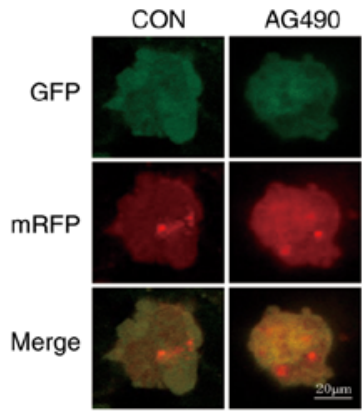

C
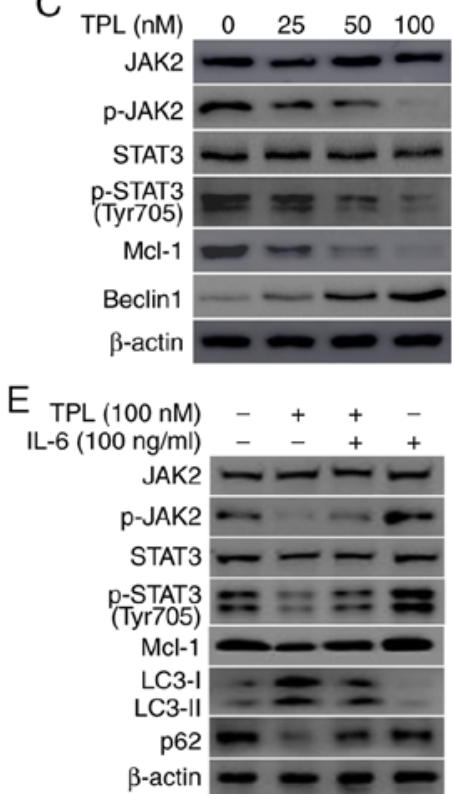
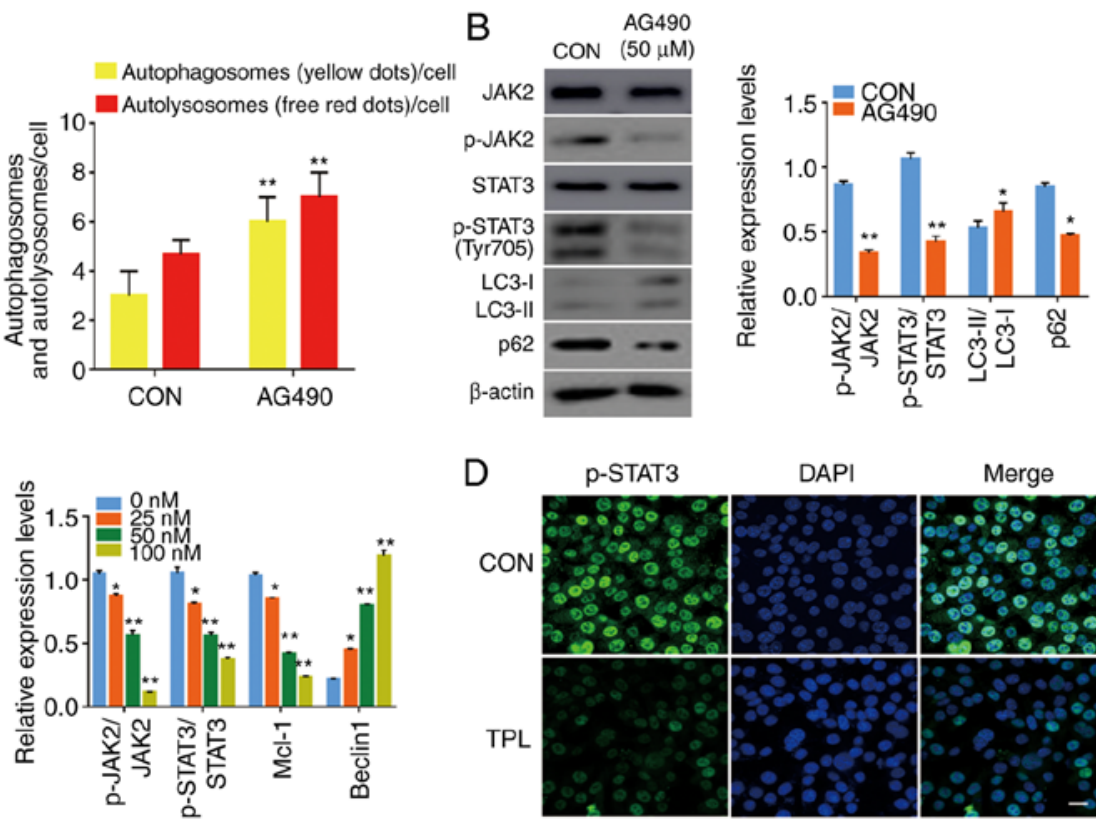

D p-STAT3


Figure 4. ROS generation is upstream of JAK2/STAT3 inactivation in TPL-induced autophagy. (A and B) SKOV3/DDP cells were treated with or without $50 \mu \mathrm{M}$ AG490 for $24 \mathrm{~h}$, and (A) the autophagosomes (yellow puncta) and the autolysosomes (red puncta) were detected using a fluorescence microscope. Scale bar, $20 \mu \mathrm{m}$. (B) The expression levels of p-JAK2, p-STAT3 (Y705), LC3 and p62 were compared by western blot analysis. (C) Western blotting revealed the expression of JAK2, p-JAK2, STAT3, p-STAT3 (Y705), Mcl-1 and Beclin1 in SKOV3/DDP cells after treatment with variable doses of TPL for 24 h. (D) SKOV3/DDP cells were treated with or without $100 \mathrm{nM}$ TPL for $24 \mathrm{~h}$, then the expression of p-STAT3 (Y705) was examined using immunofluorescence staining (magnification, $\mathrm{x} 400$ ). The cell nuclei were stained with DAPI (blue). Scale bar, $20 \mu \mathrm{m}$. (E) SKOV3/DDP cells were incubated without or with TPL (100 nM) in the presence or absence of IL-6 (100 ng/ml) for $24 \mathrm{~h}$. Mcl-1, LC3 and p62 were detected by western blot analysis. (F) SKOV3/DDP cells were treated with NAC (5 mM) for $1 \mathrm{~h}$ prior to exposure to TPL $(100 \mathrm{nM})$ for $24 \mathrm{~h}$, and then the p-JAK2 and p-STAT3 (Y705) expression were evaluated with western blotting. * P<0.05 and ${ }^{* *} \mathrm{P}<0.01$ vs. the Con group. ${ }^{\#} \mathrm{P}<0.05$ and ${ }^{\# \#} \mathrm{P}<0.01$ vs. the TPL group. ROS, reactive oxygen species; JAK2, Janus kinase 2; STAT3, signal transducer and activator of transcription-3; TPL, triptolide; DDP, cisplatin; p-phosphorylated; Mcl-1, myeloid cell leukemia-1; IL, interleukin; NAC, N-acetyl-1-cysteine; Con, Control.

\section{Discussion}

Recent studies have revealed that numerous anticancer drugs, including TPL, can concurrently activate autophagy, as they induce apoptotic cell death in tumor cells (32-34). Over the last several years, our prior studies have demonstrated the antitumor and sensitization properties of TPL against epithelial ovarian cancer in vitro and in vivo, and apoptosis has been revealed to mediate this process (9-13). However, the exact role of TPL-induced autophagy in DDP-resistant human epithelial ovarian cancer cells remains unclear. Thus, DDP-resistant human ovarian cancer SKOV3/DDP cells were used as an experimental model to address this issue. The present study demonstrated for the first time, to the best of our knowledge, that TPL induced lethal autophagy in SKOV3/DDP cells via the ROS/JAK2/STAT3 pathway (Fig. 6).

Autophagy and apoptosis are distinct cellular processes that are closely interrelated (35). In the present study, the data clearly revealed that TPL strongly elicited typical autophagic features in cells, as evidenced by the altered expression of autophagic proteins, such as LC3-II, p62 and Beclin1, and the increased numbers of autophagosomes and autolysosomes. Usually, normal cells have a low basal level of autophagy to maintain homeostasis and suppress tumor formation (36). On the other hand, a certain basal autophagic flux is necessary for highly-proliferating tumor cells to ensure metabolic requirements (37). Following chemotherapy, the basally-enhanced autophagic flux is generally regarded as an adaptive survival response that mediates tumor chemoresistance (37). However, extended autophagic flux above the optimal survival limit may have cytotoxic effects on cancer cells and ultimately facilitate cell death $(38,39)$. Clearly, inducing autophagic cell death could potentially be used as a novel strategy to manage resistant tumors (40). Further in vitro experiments revealed that pretreatment with the autophagy inhibitors 3-MA or CQ significantly abolished the inhibitory effect of TPL on cell proliferation. Consistently, it was observed that TPL treatment effectively 

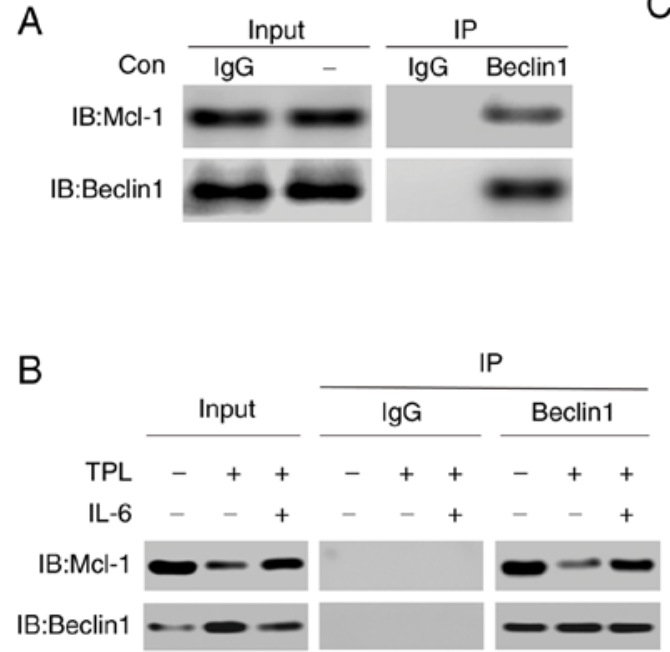
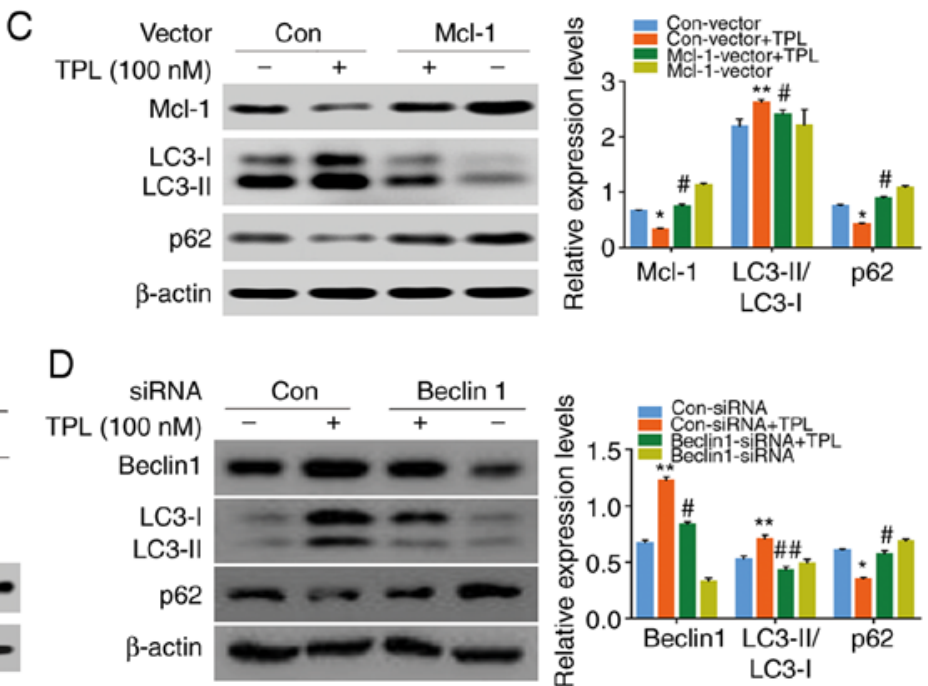

Figure 5. JAK2/STAT3 inactivation contributes to TPL-induced autophagy via regulation of Beclin1/Mcl-1 interaction. (A) Coimmunoprecipitation analysis of Beclin1 and Mcl-1 in SKOV3/DDP cells. (B) SKOV3/DDP cells were treated with TPL (100 nM) in the absence or presence of IL-6 (100 ng/ml) for $24 \mathrm{~h}$. Cell lysates were subjected to immunoprecipitation with anti-Beclin1 antibody and interaction with Mcl-1 was determined by western blotting. (C) SKOV3/DDP cells transfected with Mcl-1 overexpression plasmid were treated with or without TPL (100 nM) for $24 \mathrm{~h}$, followed by western blot analysis to detect the levels of Mcl-1, LC3 and p62. (D) SKOV3/DDP cells transfected with the Beclin1 siRNA or negative control siRNA were left cultured with or without TPL (100 nM) for $24 \mathrm{~h}$, and then the amount of Beclin1, LC3 and p62 were analyzed by western blotting. ${ }^{*} \mathrm{P}<0.05$ and ${ }^{* *} \mathrm{P}<0.01$ vs. the Con-vector group or Con-siRNA group. ${ }^{\#} \mathrm{P}<0.05$ and ${ }^{\# \#} \mathrm{P}<0.01$ vs. the Con-vector + TPL group or Con-siRNA + TPL group. JAK2, Janus kinase 2; STAT3, signal transducer and activator of transcription-3; TPL, triptolide; Mcl-1, myeloid cell leukemia-1; DDP, cisplatin; IL, interleukin; Con, Control.

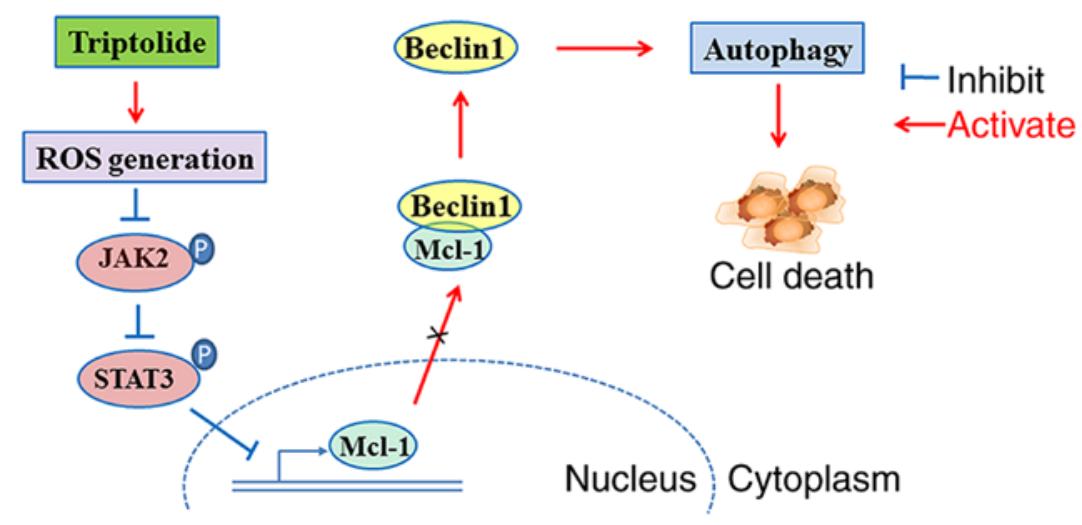

Figure 6. Schematic diagram of proposed molecular mechanism of TPL-elicited autophagy in DDP-resistant human ovarian cancer SKOV3/DDP cells. TPL treatment induces ROS generation, which can suppress the JAK2/STAT3 signaling cascade and reduce Mcl-1 expression, thereby alleviating Mcl-1/Beclin1 interaction. As a result, TPL induces Beclin1-mediated autophagy to facilitate SKOV3/DDP cell death. TPL, triptolide; DDP, cisplatin; ROS, reactive oxygen species; JAK2, Janus kinase 2; STAT3, signal transducer and activator of transcription-3; Mcl-1, myeloid cell leukemia-1.

enhanced the growth-inhibiting effect of DDP by enhancing autophagic activity in the SKOV3/DDP xenograft model. These data strongly indicated that enhanced autophagy may operate as a death mechanism in TPL-treated DDP-resistant SKOV3/DDP cells. It is highly likely that the extent of autophagic flux induced by TPL in SKOV3/DDP cells exceeds the lethal autophagic flux threshold. These observations were consistent with those of previous studies showing that TPL induced autophagy to induce apoptosis and inhibit angiogenesis in human osteosarcoma MG63 cells (26), and neuroblastoma SH-SY5Y cells (25). Some studies have reported that protective autophagy is enhanced by TPL in malignant glioma U251 cells (27) and cervical cancer SiHa cells (28). Accumulating evidence suggests that the level of basal autophagic flux and lethal autophagic flux threshold, which play a crucial role in governing cell fate, may vary according to the different tumor tissue cell types (37). These disparate effects may be, in part, attributed to the differences in tumor types and lack of a standard test method for autophagic flux threshold (37,38). Future studies will be required to delineate these questions.

Although TPL has been demonstrated to exert potent autophagic-regulating effects via different mechanisms (14), the exact molecular mechanism remains poorly understood. Previously, our group demonstrated that TPL triggered the ROS-dependent activation of apoptotic cell death in DDP-resistant ovarian cancer cells (12). ROS are highly reactive oxygen-derived molecules that influence cell survival, autophagy and apoptosis through redox signaling (29). Usually, cancer cells exhibit increased ROS generation that may promote cell survival and drug resistance (41). However, 
an excessively high level of ROS may cause excessive oxidative stress and lead to cell death through apoptosis, autophagy and necrosis (42). Novel anticancer agents have been developed to generate ROS and trigger oxidative stress-induced tumor cell death $(42,43)$. This finding suggests that ROS may play an important role in TPL-mediated autophagy induction in SKOV3/DDP cells. Consistent with our hypothesis, pretreatment with the antioxidant NAC substantially inhibited ROS generation and rescued TPL-induced autophagy, as supported by our results revealing that NAC markedly blocked TPL-induced autophagosome/autolysosome formation, LC3 conversion and p62 degradation in SKOV3/DDP cells. These data were consistent with those of previous studies which revealed that costunolide and cinobufagin induced autophagy in cancer cells through ROS production $(33,44)$.

The JAK2/STAT3 pathway is one of the well-known pathways associated with ovarian tumor growth and chemoresistance $(21,22)$. Accumulating evidence indicates that STAT3 is an important regulator of autophagy (20). Upon stimulation, STAT3 is activated and phosphorylated, and then STAT3 transfers into the nucleus to regulate the transcription of various genes, including certain autophagy-associated genes (20). Consistently, the present results revealed that p-JAK2 and p-STAT3 (Y705) were overexpressed in SKOV3/DDP cells. Notably, treatment with AG490 strongly enhanced the level of autophagy in SKOV3/DDP cells, suggesting that inhibition of the JAK2/STAT3 pathway is involved in the regulation of autophagy. It is intriguing that activated nuclear STAT3 can upregulate Mcl-1 expression, which leads to autophagy inhibition (45). A previous study by Tai et al (24) revealed that sorafenib downregulated STAT3 activity and Mcl-1 expression and induced autophagy in multiple HCC cell lines. In contrast, Mcl-1 overexpression abrogated the effect of sorafenib on autophagy. Furthermore, Mcl-1 has been reported to negatively regulate the autophagy induction through binding to Beclin1. In accordance with these findings, the present results revealed that TPL suppressed the JAK2/STAT3 signaling pathway and further downregulated Mcl-1, along with the subsequent reduction in the Beclin1/Mcl-1 interaction. These inhibitory effects of TPL could be strongly reversed by the JAK2/STAT3 signaling activator IL-6. Moreover, ectopic expression of Mcl-1 or genetic knockdown of Beclin1 impaired the autophagy-inducing effect of TPL. Thus, it is reasonable to conclude that TPL triggered Beclin1-dependent autophagy in SKOV3/DDP cells through inhibition of the JAK2/STAT3/Mcl-1 signaling cascade and disruption of the Beclin1/Mcl-1 interaction. Notably, it was further revealed that the generation of ROS by TPL precedes the inhibition of the JAK2/STAT3 pathway. It is well documented that ROS influence the enzymatic activity of JAK 2 by oxidative modification of specific cysteine residues on JAK2 $(30,46)$. The mechanism by which ROS are involved in the regulation of the JAK2/STAT3 pathway requires further study.

Collectively, it was proposed that TPL induced ROS generation to suppress the JAK2/STAT3 signaling cascade, causing a decline in Mcl-1 expression and alleviating Mcl-1-mediated inhibition of Beclin1, consequently triggering autophagic cell death in SKOV3/DDP cells (Fig. 6). In addition, TPL enhanced the sensitivity of SKOV3/DDP cells to DDP by increasing the autophagic response in vivo. The present results not only deepen the understanding of the molecular mechanism of
TPL-mediated antitumor effects but also support the use of TPL as a therapeutic option for patients with resistant ovarian tumors in the future.

\section{Acknowledgements}

Not applicable.

\section{Funding}

The present study was supported by the National Natural Science Foundation of China (grant no. 81760729), the Project of the Jiangxi Provincial Natural Science Foundation in China (grant no. 20202BABL206102), the Project of the Jiangxi Provincial Health Commission (grant no. 20204271), the Youth Science Foundation of Science and Technology Program of the Second Affiliated Hospital of Nanchang University (grant no. 2019YNQN12001).

\section{Availability of data and materials}

The datasets used during the present study are available from the corresponding author upon reasonable request.

\section{Author's contributions}

BT and YZ conceived and designed the present study. FL, YZ, JC, CL, XZ, XW, FX, QZ performed the experiments. YZ, CL, FL, XW, JC, XZ, FX, QZ and BT performed the data acquisition and analysis. YZ wrote the manuscript. YZ, CL, XW, FL and BT revised the work critically for important intellectual content. All the authors read and approved the final manuscript.

\section{Ethics approval and consent to participate}

All animal experiments were approved by the Ethics Committee of Nanchang University (Nanchang, China) and performed in compliance with the approved guidelines.

\section{Patient consent for publication}

Not applicable.

\section{Competing interests}

The authors declare that they have no competing interests.

\section{References}

1. Lheureux S, Gourley C, Vergote I and Oza AM: Epithelial ovarian cancer. Lancet 393: 1240-1253, 2019.

2. Orr B and Edwards RP: Diagnosis and treatment of ovarian cancer. Hematol Oncol Clin North Am 32: 943-964, 2018.

3. Armbruster S, Coleman RL and Rauh-Hain JA: Management and treatment of recurrent epithelial ovarian cancer. Hematol Oncol Clin North Am 32: 965-982, 2018.

4. Pignata S, Pisano C, Di Napoli M, Cecere SC, Tambaro R and Attademo L: Treatment of recurrent epithelial ovarian cancer. Cancer 125 (Suppl 24): S4609-S4615, 2019.

5. Wang H, Ma D, Wang C, Zhao S and Liu C: Triptolide inhibits invasion and tumorigenesis of hepatocellular carcinoma MHCC-97H cells through NF-кB Signaling. Med Sci Monit 22: 1827-1836, 2016. 
6. Kim ST, Kim SY, Lee J, Kim K, Park SH, Park YS, Lim HY, Kang WK and Park JO: Triptolide as a novel agent in pancreatic cancer: The validation using patient derived pancreatic tumor cell line. BMC Cancer 18: 1103, 2018.

7. Wang T, Ding Y, Yang Y, Wang Z, Gao W, Li D, Wei J and Sun Y: Synergistic antitumour effects of triptolide plus 10-hydroxycamptothecin onbladder cancer. Biomed Pharmacother 115: 108899, 2019.

8. Liu H, Tang L, Li X and Li H: Triptolide inhibits vascular endothelial growth factor-mediated angiogenesis in human breast cancer cells. Exp Ther Med 16: 830-836, 2018

9. Hu H, Luo L, Liu F, Zou D, Zhu S, Tan B and Chen T: Anti-cancer and sensibilisation effect of triptolide on human epithelial ovarian cancer. J Cancer 7: 2093-2099, 2016.

10. Hu H, Huang G, Wang H, Li X, Wang X, Feng Y, Tan B and Chen T: Inhibition effect of triptolide on human epithelial ovarian cancer via adjusting cellular immunity and angiogenesis Oncol Rep 39: 1191-1196, 2018.

11. Huang G, Hu H, Zhang Y, Zhu Y, Liu J, Tan B and Chen T: Triptolide sensitizes cisplatin-resistant human epithelial ovarian cancer by inhibiting the phosphorylation of AKT. J Cancer 10: 3012-3020, 2019

12. Zhong YY, Chen HP, Tan BZ, Yu HH and Huang XS: Triptolide avoids cisplatin resistance and induces apoptosis via the reactive oxygen species/nuclear factor- $\kappa \mathrm{B}$ pathway in SKOV3 ${ }^{\mathrm{PT}}$ platinum-resistant human ovarian cancer cells. Oncol Lett 6 : 1084-1092, 2013

13. Hu H, Zhu S, Tong Y, Huang G, Tan B and Yang L: Antitumor activity of triptolide in SKOV3 cells and SKOV3/DDP in vivo and in vitro. Anticancer Drugs 31: 483-491, 2020.

14. Wei YM, Wang YH, Xue HQ, Luan ZH, Liu BW and Ren JH: Triptolide, A potential autophagy modulator. Chin J Integr Med 25: 233-240, 2019.

15. Kocaturk NM, Akkoc Y, Kig C, Bayraktar O, Gozuacik D and Kutlu O: Autophagy as a molecular target for cancer treatment. Eur J Pharm Sci 134: 116-137, 2019.

16. Poillet-Perez L and White E: Role of tumor and host autophagy in cancer metabolism. Genes Dev 33: 610-619, 2019.

17. Denton D and Kumar S: Autophagy-dependent cell death. Cell Death Differ 26: 605-616, 2019.

18. Pietrocola F, Izzo V, Niso-Santano M, Vacchelli E, Galluzzi L, Maiuri MC and Kroemer G: Regulation of autophagy by stress-responsive transcription factors. Semin Cancer Biol 23: 310-322, 2013

19. Hao D, Wen X, Liu L, Wang L, Zhou X, Li Y, Zeng X, He G and Jiang X: Sanshool improves UVB-induced skin photodamage by targeting JAK2/STAT3-dependent autophagy. Cell Death Dis 10: 19, 2019.

20. You L, Wang Z, Li H, Shou J, Jing Z, Xie J, Sui X, Pan H and Han W: The role of STAT3 in autophagy. Autophagy 11: 729-739, 2015.

21. Yoshikawa T, Miyamoto M, Aoyama T, Soyama H, Goto T Hirata J, Suzuki A, Nagaoka I, Tsuda H, Furuya K and Takano M: JAK2/STAT3 pathway as a therapeutic target in ovarian cancers. Oncol Lett 15: 5772-5780, 2018.

22. Gritsina G, Xiao F, O'Brien SW, Gabbasov R, Maglaty MA, $\mathrm{Xu}$ RH, Thapa RJ, Zhou Y, Nicolas E, Litwin S, et al: Targeted blockade of JAK/STAT3 signaling inhibits ovarian carcinoma growth. Mol Cancer Ther 14: 1035-1047, 2015.

23. Zheng X, Chen W, Hou H, Li J, Li H, Sun X, Zhao L and Li X: Ginsenoside 20(S)-Rg3 induced autophagy to inhibit migration and invasion of ovarian cancer. Biomed Pharmacother 85 620-626, 2017

24. Tai WT, Shiau CW, Chen HL, Liu CY, Lin CS, Cheng AL, Chen PJ and Chen KF: Mcl-1-dependent activation of Beclin 1 mediates autophagic cell death induced by sorafenib and SC-59 in hepatocellular carcinoma cells. Cell Death Dis 4: e485, 2013.

25. Krosch TC, Sangwan V, Banerjee S, Mujumdar N, Dudeja V, Saluja AK and Vickers SM: Triptolide-mediated cell death in neuroblastoma occurs by both apoptosis and autophagy pathways and results in inhibition of nuclear factor-kappa B activity. Am J Surg 205: 387-396, 2013.

26. Li X, Lu Q, Xie W, Wang Y and Wang G: Anti-tumor effects of triptolide on angiogenesis and cell apoptosis in osteosarcoma cells by inducing autophagy via repressing Wnt/ $\beta$-Catenin signaling. Biochem Biophys Res Commun 496: 443-449, 2018.
27. Liu X, Zhao P, Wang X, Wang L, Zhu Y and Gao W: Triptolide induces glioma cell autophagy and apoptosis via upregulating the ROS/JNK and downregulating the Akt/mTOR signaling pathways. Front Oncol 9: 387, 2019.

28. Qin G, Li P and Xue Z: Triptolide induces protective autophagy and apoptosis in human cervical cancer cells by downregulating Akt/mTOR activation. Oncol Lett 16: 3929-3934, 2018.

29. Kaminskyy VO and Zhivotovsky B: Free radicals in cross talk between autophagy and apoptosis. Antioxid Redox Signal 21: 86-102, 2014

30. Cao Y, Wang J, Tian $\mathrm{H}$ and Fu GH: Mitochondrial ROS accumulation inhibiting JAK2/STAT3 pathway is a critical modulator of CYT997-induced autophagy and apoptosis in gastric cancer. J Exp Clin Cancer Res 39: 119, 2020.

31. Germain M, Nguyen AP, Le Grand JN, Arbour N, Vanderluit JL, Park DS, Opferman JT and Slack RS: MCL-1 is a stress sensor that regulates autophagy in a developmentally regulated manner. EMBO J 30: 395-407, 2011.

32. Gali-Muhtasib H, Hmadi R, Kareh M, Tohme R and Darwiche N: Cell death mechanisms of plant-derived anticancer drugs: Beyond apoptosis. Apoptosis 20: 1531-1562, 2015.

33. Ma K, Zhang C, Huang MY, Li WY and Hu GQ: Cinobufagin induces autophagy-mediated cell death in human osteosarcoma U2OS cells through the ROS/JNK/p38 signaling pathway. Oncol Rep 36: 90-98, 2016.

34. Chang CH, Lee CY, Lu CC, Tsai FJ, Hsu YM, Tsao JW, Juan YN, Chiu HY, Yang JS and Wang CC: Resveratrol-induced autophagy and apoptosis in cisplatin-resistant human oral cancer CAR cells: A key role of AMPK and Akt/mTOR signaling. Int J Oncol 50 873-882, 2017.

35. Cooper KF: Till death do us part: The marriage of autophagy and apoptosis. Oxid Med Cell Longev 2018: 4701275, 2018.

36. Fitzwalter BE and Thorburn A: Recent insights into cell death and autophagy. FEBS J 282: 4279-4288, 2015.

37. Bhat P, Kriel J, Shubha Priya B, Basappa, Shivananju NS and Loos B: Modulating autophagy in cancer therapy: Advancements and challenges for cancer cell death sensitization. Biochem Pharmacol 147: 170-182, 2018.

38. Kriel J and Loos B: The good, the bad and the autophagosome: Exploring unanswered questions of autophagy-dependent cell death. Cell Death Differ 26: 640-652, 2019.

39. Fulda S and Kögel D: Cell death by autophagy: Emerging molecular mechanisms and implications for cancer therapy. Oncogene 34: 5105-5113, 2015.

40. Cirone M, Gilardini Montani MS, Granato M, Garufi A, Faggioni A and D'Orazi G: Autophagy manipulation as a strategy for efficient anticancer therapies: Possible consequences. J Exp Clin Cancer Res 38: 262, 2019.

41. Galadari S, Rahman A, Pallichankandy S and Thayyullathil F: Reactive oxygen species and cancer paradox: To promote or to suppress? Free Radic Biol Med 104: 144-164, 2017.

42. Zou Z, Chang H, Li H and Wang S: Induction of reactive oxygen species: An emerging approach for cancer therapy. Apoptosis 22: $1321-1335,2017$

43. Gao L, Loveless J, Shay C and Teng Y: Targeting ROS-Mediated crosstalk between autophagy and apoptosis in cancer. Adv Exp Med Biol 1260: 1-12, 2020.

44. Fang Y, Li J, Wu Y, Gui J and Shen Y: Costunolide inhibits the growth of OAW42-A multidrug-resistant human ovarian cancer cells by activating apoptotic and autophagic pathways, production of reactive oxygen species (ROS), cleaved caspase-3 and cleaved caspase-9. Med Sci Monit 25: 3231-3237, 2019.

45. Ma Z, Bao X and Gu J: Furowanin A-induced autophagy alleviates apoptosis and promotes cell cycle arrest via inactivation STAT3/Mcl-1 axis in colorectal cancer. Life Sci 218: 47-57, 2019.

46. Sun Q, Lu NN and Feng L: Apigetrin inhibits gastric cancer progression through inducing apoptosis and regulating ROS-modulated STAT3/JAK2 pathway. Biochem Biophys Res Commun 498: 164-170, 2018

This work is licensed under a Creative Commons Attribution-NonCommercial-NoDerivatives 4.0 International (CC BY-NC-ND 4.0) License. 